\title{
Efeitos da Drenagem Linfática Manual na Diminuição do Edema de Membros Inferiores em Gestantes
}

\section{Effects of Manual Lymphatic Drainage on the Reduction of Lower Limb Edema in Pregnant Women}

Alexandre Delgado ${ }^{1}$, Catarina Rattes ${ }^{2}$, Maria Eduarda Soathman de Abreu ${ }^{3}$, Mikaela Aparecida de Oliveira Xavier ${ }^{3}$, Diego Luis de Queiroz Araújo ${ }^{3}$, Rita de Cassia Barros da Silva ${ }^{3}$, Iza Paula de Deus Mello Albuquerque Arruda ${ }^{4}$ e Marcelo Renato Guerino ${ }^{5}$.

1. Professor Mestre do Curso de Fisioterapia, Centro Universitário Escritor Osman da Costa Lins (UNIFACOL). Vitória de Santo Antão, PE. Doutorando em Saúde Integral pela Instituto de Medicina Integral Prof. Fernando Figueira - IMIP.

2. Professora Doutora do curso de Fisioterapia (UNIFACOL).

3. Graduando(a) do Curso de Fisioterapia (UNIFACOL).

4. Professora e Coordenadora do Curso de Fisioterapia (UNIFACOL).

5. Professor Doutor do Departamento de Fisioterapia da Universidade Federal de Pernambuco (UFPE).

alexmagno_d@hotmail.com e marceloguerino@hotmail.com.

\section{Palavras-chave}

Gestação

Fisioterapia

Edema

\section{Keywords}

Gestation

Physiotherapy

Edema

\begin{abstract}
Resumo: Avaliar os efeitos da drenagem linfática na prevenção e diminuição do edema de membros inferiores em gestantes no segundo e terceiro trimestre gestacional. Trata-se de uma série de caso. A população foi composta por 10 gestantes no segundo e terceiro trimestre gestacional. A drenagem linfática foi realizada com a paciente em decúbito dorsal, a 45 graus. Foi iniciada com a estimulação na região cervical por 15 minutos, em seguida estímulos nos linfonodos inguinais de ambos os membros inferiores, poplíteo e maleolar. Cada membro inferior foi drenado durante 20 minutos. Houve redução do edema nos membros inferiores das gestantes em média de dois centímetros logo após a primeira sessão (efeito agudo) e após a última sessão (efeito tardio) com redução de até cinco centímetros. Houve diferença significativa tanto no efeito agudo quanto no efeito tardio em relação à dor e a fadiga nos membros inferiores. Todas as gestantes ficaram satisfeitas e recomendariam o tratamento. A técnica de drenagem linfática manual se mostrou eficiente na prevenção e diminuição do edema, dor e fadiga nos membros inferiores em fase aguda e no efeito tardio. Todas as gestantes ficaram satisfeitas com a drenagem linfática e recomendariam o tratamento.

Abstract: To evaluate the effects of lymphatic drainage on the prevention and reduction of patrimonial deficit in the second and third gestational trimesters. This is a series of cases. The collection was composed of 10 pregnant women in the second and third gestational trimesters. Lymphatic drainage was performed with one patient in dorsal decubitus at 45 degrees. Initiative cervical for 15 minutes, then there are stimuli in the inguinal lymph nodes of both lower limbs, popliteal and malleolar. Each lower limb was drained for 20 minutes. The reduction of edema occurred in the water drops of the pregnant women, averaging two centimeters shortly after a first session (acute effect) and after a last session (late reduction) with a reduction of five centimeters. The last memory in the object of the last lower object in the last below. All the pregnant women were satisfied and recommended the treatment. The manual lymphatic drainage technique is based on the prevention and reduction of edema, pain and fatigue in the lower limbs in the acute phase and without late effect. All the pregnant women were satisfied with the lymphatic drugstore and recommended the treatment.
\end{abstract}

Artigo recebido em: 12.07.2019

Aprovado para publicação em: 07.08.2019
DELGADO, A. et al. Efeitos da Drenagem Linfática Manual na Diminuição do Edema de Membros Inferiores em Gestantes.
Pleiade, 13(28): 49-59, Jan./Jun., 2019

DOI: 10.32915/pleiade.v13i28.545 


\section{INTRODUÇão}

O segundo trimestre gestacional é o período que compreende da décima quarta até a vigésima sexta semana $\left(14^{\mathrm{a}}-26^{\mathrm{a}}\right)$ e o terceiro trimestre gestacional é compreendido no período da vigésima sétima até a quadragésima semana $\left(27^{\mathrm{a}}-40^{\mathrm{a}}\right)$ e é nesse período que surgem mudanças significativas no corpo da mulher. $\mathrm{O}$ aumento da produção hormonal é considerado o fator principal de tais características, fazendo com que ocorra um aumento do peso corporal da gestante (FONSECA et al., 2009).

No terceiro trimestre os incômodos aparecem com mais intensidade, gerando desconfortos para as gestantes que apresentam um sinal bastante frequente de edema dos membros inferiores. O edema gestacional é o acúmulo excessivo de líquido intersticial nos espaços intercelulares, dificultando a permeabilidade capilar (LEDUC e LEDUC, 2000; GUIRRO e GUIRRO, 2003).

Os fatores que colaboram para a formação do edema na gestação é o aumento da permeabilidade capilar, aumento da pressão capilar, compressão de válvulas nervosas, diminuição na quantidade de proteínas no sangue e retenção de líquido que ocorre em virtude dos hormônios estrogênio, cortisol, progesterona e relaxina (SOUZA, 2000). O edema apresenta sensações desconfortáveis para a mulher no período gestacional e sintomatologias como a dor, fadiga, sensação de peso e dormência nos pés e nas pernas são frequentes, além de ser um fator que pode contribuir na estética afetando a autoestima da gestante (BAMIGBOYE e HOFMEYR, 2006).

Entre os tratamentos mais apropriados para prevenir e tratar o edema durante a gestação, a drenagem lin fática manual (DLM) está entre as mais utilizadas (WOLF e THEISS). A pressão mecânica da massagem manual elimina o acumulo de líquidos e diminui as chances da formação de fibrose, fazendo a expulsão do líquido do meio tissular para os vasos linfáticos e venosos, dessa maneira mantendo o equilíbrio hídrico dos espaços intersticiais das células. O corpo fisiologicamente drena os líquidos através do sistema linfático, po rém se porventura algum fator interno alterar, e essa função se torne insuficiente é necessário um auxílio ex terno que pode ser realizada por quatro maneiras distintas: manual, corrente galvânica, eletroestimulação sequencial e pressoterapia. Das quatro, acredita-se que a mais eficaz é a realizada manualmente (LEDUC e LEDUC, 2000; GUIRRO e GUIRRO, 2003).

A DLM adere movimentos superficiais, realizadas em ritmo contínuo e lento para que a linfa seja levada gradualmente, de maneira harmônica ao movimento natural (EMRICH, 2013). Geralmente inicia-se com a técnica de “evacuação” ou "desbloqueio” das áreas proximais do membro comprometido fazendo o movimento de bombeamento. Depois segue para as regiões distais, aumentando a motricidade do "linfangion”, responsável por carregar a linfa, e desta forma aumentando também o fluxo do sistema linfático (SANTOS, 2009)

A técnica da DLM promove benefício à gestante, pois aumenta a capacidade de locomoção da linfa no interior dos gânglios linfáticos ajudando na eliminação dos líquidos, dessa maneira, reduzindo o edema (LE DUC e LEDUC, 2000). Outro fator crucial além da eliminação do edema é a prevenção, porque evita a desidratação, especialmente nas gestantes que tem predisposição a retenção de líquidos, auxiliando também na troca de nutrientes dos tecidos (SILVA et al., 2015).

Diante do exposto, o objetivo do estudo foi avaliar os efeitos da drenagem linfática na prevenção e diminuição do edema de membros inferiores em gestantes no segundo e terceiro trimestre gestacional. 


\section{Material e Métodos}

Trata-se de um estudo do tipo série de casos realizados na FACOL - Faculdade Escritor Osman da Costa Lins, localizada no município de Vitória de Santo Antão - PE. A coleta dos dados foi realizada no período compreendido entre os meses de março a maio de 2018. A população foi composta por gestantes no segundo e terceiro trimestre gestacional que preencheram aos critérios de elegibilidade. A amostra foi composta por 10 gestantes obtidas de forma sequencial por conveniência.

Esta pesquisa foi desenvolvida conforme as recomendações da Resolução n ${ }^{0}$ 466/12, do Conselho Nacional de Saúde do Ministério da Saúde, que dispõem sobre as pesquisas envolvendo seres humanos, após a aprovação do Comitê de Ética em Pesquisa da Fundação de Hematologia e Hemoterapia do Estado de Per nambuco - HEMOPE, sob o ${ }^{\circ}$ do Processo: 2.650.349.

Como critério de inclusão foram utilizados os seguintes parâmetros: idade de 18 a 35 anos; gestantes a partir da décima quarta semana gestacional; primípara e/ou Multípara e gestação de feto único; e como critérios de exclusão gestantes com alguma instabilidade clínica; gestantes com Hipertensão não controlada; ges tantes com insuficiência renal e gestantes com trombose venosa Profunda.

\section{Coleta de Dados}

Antes do início do tratamento, foi coletada a assinatura do Termo de Consentimento Livre e Esclarecido (TCLE) das gestantes, autorizando a participação da mesma no estudo, os quais descreviam todos os procedimentos realizados na pesquisa, assim como seus riscos e desconfortos, benefícios e garantia de confiabilidade de seus dados. Para coleta de dados, foi desenvolvido um formulário de avaliação para esta pesquisa, composto por dados clínicos, obstétricos e sociodemográficos que foi aplicada em forma de entrevista.

A avaliação do edema gestacional dos membros inferiores foi realizada pela perimetria, utilizando uma fita métrica, antes e depois da drenagem linfática manual. Foram mensurados dez níveis de perimetria nos membros inferiores: A partir da linha poplítea como marco zero, em direção à coxa e à perna, medindo-se a cada $7 \mathrm{~cm}$, e uma medida específica do tornozelo (ESTERSON, 1979).

Para avaliar a dor e a fadiga dos membros inferiores foi utilizada a Escala Visual Analógica (EVA), com preendida como uma linha horizontal de $10 \mathrm{~cm}$ com as extremidades indicando "ausência de dor" e "a pior dor possível”. Foi considerado dor e fadiga intensa igual ou maior que 8, moderada entre 4 e 7 e leve 1 a 3 (PIMENTA, 1994).

Foram realizadas sessões de drenagem linfática manual, duas vezes por semana por 60 minutos. As ses sões da DLM foram finalizadas após a paciente entrar em trabalho de parto e parir. Foram aferidos os sinais vitais e a pressão arterial, antes e após os procedimentos.

A drenagem linfática foi realizada com a paciente em decúbito dorsal, a 45 graus (FIGURA 1). Inicialmente foi realizado a estimulação na região cervical por 15 minutos (FIGURA 2), logo em seguida foram fei tos estímulos nos linfonodos inguinais de ambos membros inferiores, poplíteo e maleolar. Cada membro inferior foi drenado durante 20 minutos. Os seguimentos de coxa e pernas foram divididos em três, e em seguida iniciou-se as manobras em deslizamento na face medial da coxa, na face anterior para medial e da face lateral em direção a medial. Com os membros inferiores levemente fletidos iniciou-se a drenagem na face posterior da coxa, com a linfa sendo drenada para toda face medial do membro inferior (FIGURA 3). 
Figura 1. Posicionamento da gestante.

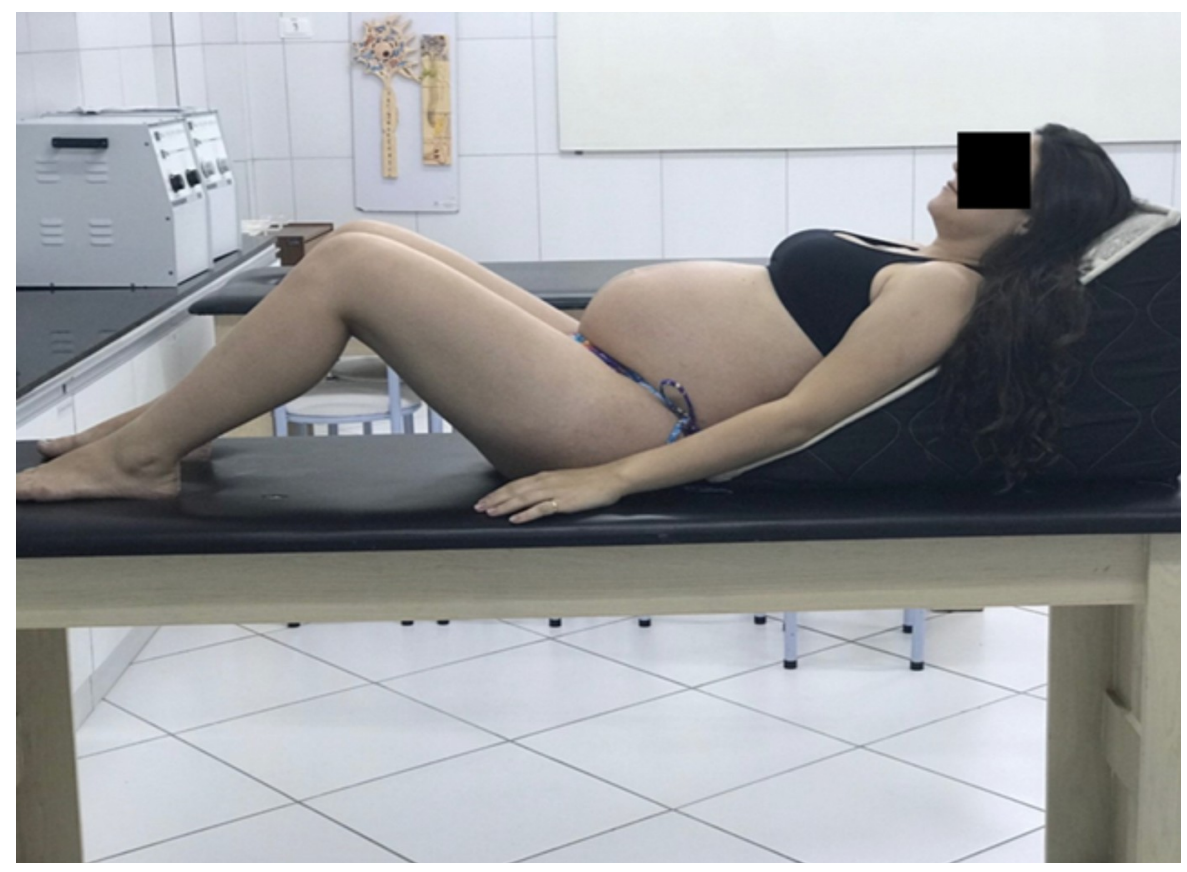

Figura 2. Estimulação da região cervical

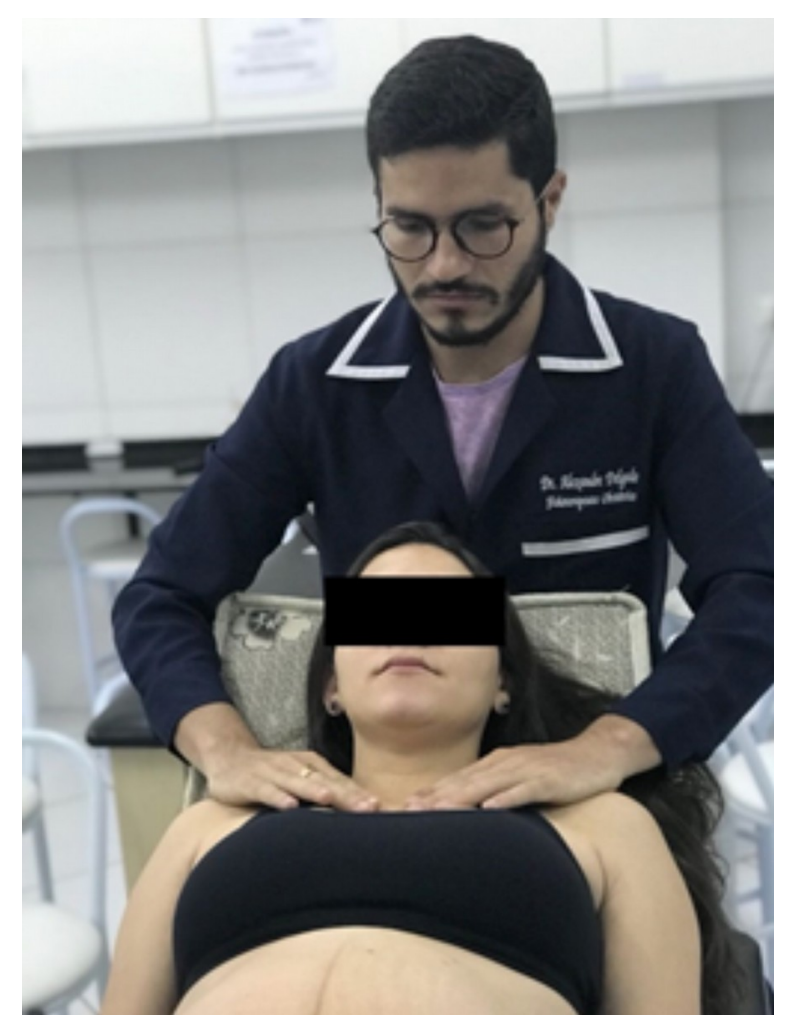


Figura 3. Sequência da DLM.

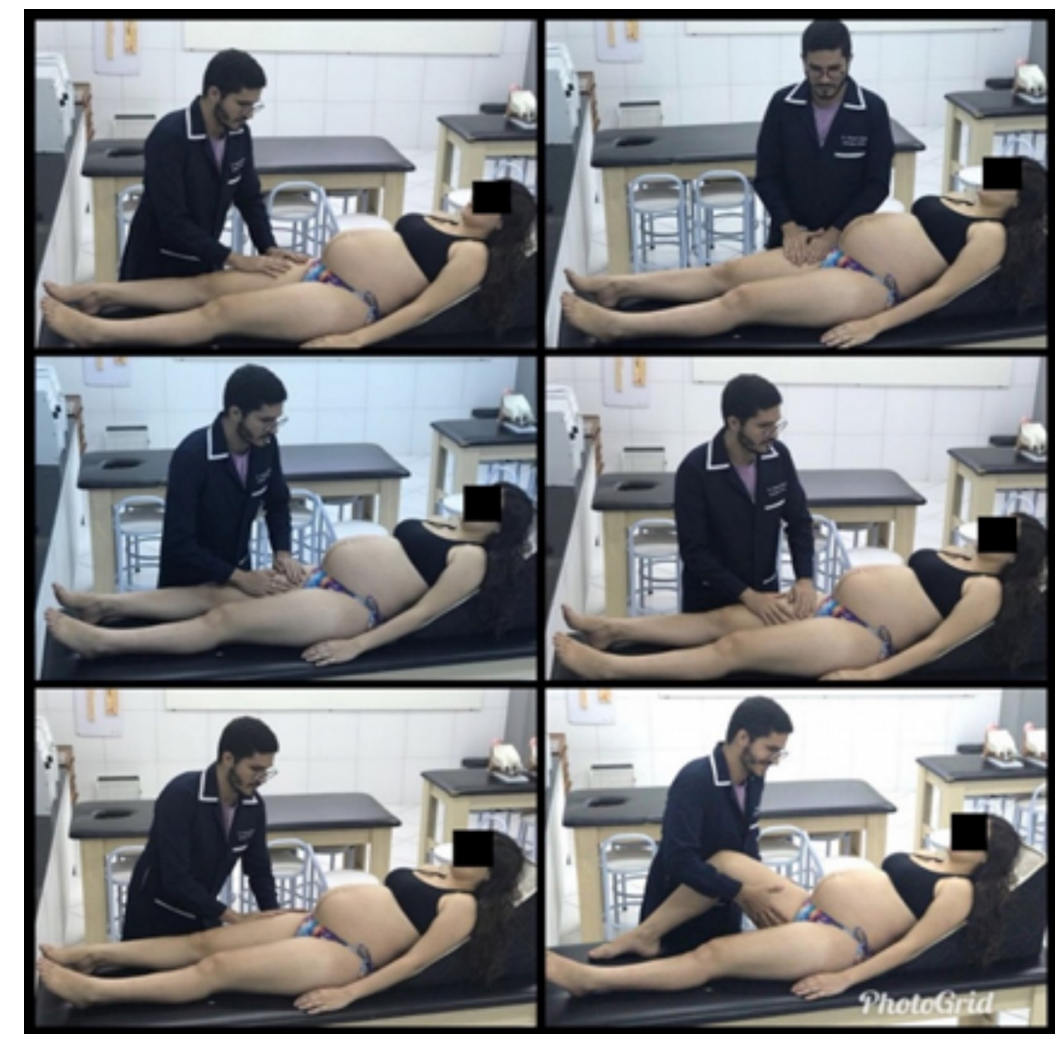

Ao final das sessões da drenagem linfática manual, foi perguntado as gestantes se elas recomendariam ou não o procedimento recebido. O nível de satisfação do tratamento foi avaliado em: (-) Insatisfeita, $(+)$ Pouco Satisfeita, (++) Moderadamente Satisfeita e (+++) Muito Satisfeita.

\section{Análise de Dados}

Na análise descritiva, os dados foram expostos em tabelas de distribuição de frequência para as variáveis categóricas e medidas de tendência central e de dispersão para as variáveis numéricas. Os dados da perimetria, dor e fadiga dos membros inferiores foram considerados para análise estatística, utilizando-se o teste $\mathrm{t}$ pareado. Os dados foram armazenados no programa SPSS versão 20.0 e em todas as etapas da análise foi considerado o nível de significância de 5\%.

\section{Resultados}

Analisando as características das participantes notou-se que a média de idade foi de 25 anos e idade ges tacional de 26 semanas. A maioria das gestantes nunca vivenciaram um parto. Mais de (70\%) eram casadas e (90\%) delas possuem alta escolaridade com mais de 12 anos de estudo. Quase todas são do interior de Per nambuco. A porcentagem foi igual no que diz respeito à ocupação, no qual (50\%) das voluntárias são do lar e (50\%) possuem outras ocupações. Mais da metade das gestantes possuíam varizes e poucas telangectasias. A maioria não fazia uso de meia elástica e 60\% delas possuíam edema (Tabela 1).

DELGADO, A. et al. Efeitos da Drenagem Linfática Manual na Diminuição do Edema de Membros Inferiores em Gestantes.
Pleiade, 13(28): 49-59, Jan./Jun., 2019 DOI: $10.32915 /$ pleiade.v13i28.545 
Tabela 1. Características das participantes. Vitória de Santo Antão - PE. 2018.

\begin{tabular}{|c|c|}
\hline Característica & Gestantes $(n=10)$ \\
\hline $\begin{array}{c}\text { Idade } \\
\text { Variação } \\
\text { Média } \pm \text { DP }\end{array}$ & $\begin{array}{c}18-35 \\
25 \pm 5.83 \text { (anos) }\end{array}$ \\
\hline $\begin{array}{c}\text { Idade Gestacional (Semana) } \\
\text { Variação } \\
\text { Média } \pm \text { DP }\end{array}$ & $\begin{array}{c}18-34 \\
26 \pm 6.04 \text { (semanas) }\end{array}$ \\
\hline $\begin{array}{c}\text { Número de Partos n (\%) } \\
\text { Nulípara } \\
\text { Primípara }\end{array}$ & $\begin{array}{l}8(80 \%) \\
2(20 \%)\end{array}$ \\
\hline $\begin{array}{c}\text { Estado Civil n (\%) } \\
\text { Solteira } \\
\text { Casada }\end{array}$ & $\begin{array}{l}3(30 \%) \\
7(70 \%)\end{array}$ \\
\hline $\begin{array}{c}\text { Escolaridade } n(\%) \\
\text { 8-11 anos de estudo } \\
\text { Mais de } 12 \text { anos de estudos }\end{array}$ & $\begin{array}{l}1(10 \%) \\
9(90 \%)\end{array}$ \\
\hline $\begin{array}{c}\text { Procedência n (\%) } \\
\text { Região Metropolitana } \\
\text { Interior de Pernambuco }\end{array}$ & $\begin{array}{l}1(10 \%) \\
9(90 \%)\end{array}$ \\
\hline $\begin{array}{l}\text { Ocupação n (\%) } \\
\text { Do Lar } \\
\text { Outras }\end{array}$ & $\begin{array}{l}5(50 \%) \\
5(50 \%)\end{array}$ \\
\hline $\begin{array}{c}\text { Presença de Varizes } \\
\text { Sim } \\
\text { Não }\end{array}$ & $\begin{array}{l}2(20 \%) \\
8(80 \%)\end{array}$ \\
\hline $\begin{array}{c}\text { Presença de Telangectasias } \\
\text { Sim } \\
\text { Não }\end{array}$ & $\begin{array}{l}1(10 \%) \\
9(90 \%)\end{array}$ \\
\hline $\begin{array}{c}\text { Presença de Edema } \\
\text { Sim } \\
\text { Não }\end{array}$ & $\begin{array}{l}6(60 \%) \\
4(40 \%)\end{array}$ \\
\hline $\begin{array}{l}\text { Uso de Meia Elástica } \\
\text { Sim } \\
\text { Não }\end{array}$ & $\begin{array}{l}1(10 \%) \\
9(90 \%)\end{array}$ \\
\hline
\end{tabular}

Por não definir a quantidade de sessões que cada gestante deveria realizar, o número de sessões variou entre elas. A maioria conseguiu realizar mais de sete sessões de drenagem linfática manual (tabela 2).

Em relação ao edema gestacional nos membros inferiores das gestantes, observou-se uma redução em média de dois centímetros da perimetria nos 10 pontos de referência de ambos os membros inferiores, logo após a primeira sessão (efeito agudo). Também houve diferença significativa após a última sessão da drena gem linfática manual no efeito tardio, com variação de redução em média do edema de 0,5 - 5,8 centímetros (Tabela 3). 
Tabela 2. Quantidade de sessões finalizadas. Vitória de Santo Antão - PE. 2018.

\begin{tabular}{cc}
\hline VOLUNTÁRIAS & NÚMERO DE SESSÕES \\
\hline 1 & 10 sessões \\
2 & 4 sessões \\
3 & 7 sessões \\
4 & 7 sessões \\
5 & 10 sessões \\
6 & 4 sessões \\
7 & 10 sessões \\
8 & 10 sessões \\
9 & 6 sessões \\
10 & 3 sessões \\
\hline
\end{tabular}

Tabela 3. Perimetria antes e após a DL dos MMII, efeito agudo e efeito tardio. Vitória de Santo Antão - PE. 2018.

\begin{tabular}{|c|c|c|c|c|c|c|c|}
\hline \multirow{2}{*}{$\begin{array}{l}\text { Perimetria dos } \\
\text { MMII } \\
\text { MIE }\end{array}$} & \multicolumn{3}{|c|}{$\begin{array}{c}\text { Efeito agudo } \\
\text { (Primeira Sessão) }\end{array}$} & \multirow{2}{*}{$\begin{array}{r}\begin{array}{r}\text { Teste-t } \\
\text { pareado }\end{array} \\
\text { P valor }\end{array}$} & \multicolumn{2}{|c|}{$\begin{array}{l}\text { Efeito crônico } \\
\text { (Última Sessão) }\end{array}$} & \multirow{2}{*}{$\begin{array}{r}\text { Teste-t } \\
\text { pareado } \\
\text { P valor }\end{array}$} \\
\hline & Pré & Pós & Dif(IC95\%) & & Pós & Dif(IC95\%) & \\
\hline Linha Inguinal & $67,8 \pm 9,3$ & $65,6 \pm 8,4$ & $2,2(1,1$ a 3,3$)$ & 0,001 & $63,9 \pm 10,4$ & $3,9(1,1$ a 6,7$)$ & 0,012 \\
\hline $7 \mathrm{~cm}$ & $62,7 \pm 9,1$ & $60,8 \pm 7,9$ & $2,0(0,5$ a 3,4$)$ & 0,016 & $59,7 \pm 9,7$ & $3,1(0,1$ a 6,1$)$ & 0,004 \\
\hline $14 \mathrm{~cm}$ & $56,6 \pm 8,3$ & $55,4 \pm 8,1$ & $1,2(0,4$ a 2,0$)$ & 0,007 & $55,8 \pm 12,4$ & $0,8(-5,1$ a 6,7$)$ & 0,766 \\
\hline $21 \mathrm{~cm}$ & $52,1 \pm 8,1$ & $50,5 \pm 7,8$ & $1,7(1,2$ a 2,1$)$ & $<0,001$ & $50,0 \pm 2,3$ & $1,9(1,0$ a 5,8$)$ & 0,009 \\
\hline Linha Poplítea & $46,6 \pm 6,3$ & $45,4 \pm 5,8$ & $1,3(0,7$ a 1,9$)$ & 0,001 & $44,1 \pm 5,3$ & $2,6(1,0$ a 4,2$)$ & 0,005 \\
\hline $7 \mathrm{~cm}$ & $37,8 \pm 4,3$ & $36,7 \pm 4,1$ & $1,1(0,4$ a 1,8$)$ & 0,006 & $37,1 \pm 3,9$ & $0,7(-0,6$ a 2,0$)$ & 0,257 \\
\hline $14 \mathrm{~cm}$ & $38,3 \pm 4,7$ & $37,0 \pm 4,5$ & $1,3(0,7$ a 1,9$)$ & $<0,001$ & $36,6 \pm 5,5$ & $1,7(0,6$ а 2,7$)$ & 0,005 \\
\hline $21 \mathrm{~cm}$ & $33,1 \pm 4,7$ & $31,7 \pm 5,0$ & $1,4(0,4$ a 2,3$)$ & 0,012 & $32,4 \pm 5,4$ & $0,7(-0,8$ a 2,2$)$ & 0,304 \\
\hline $28 \mathrm{~cm}$ & $28,3 \pm 3,7$ & $26,9 \pm 3,4$ & $1,4(1,0$ a 1,8$)$ & $<0,001$ & $27,0 \pm 4,2$ & $1,3(-0,4 a$ 12,9) & 0,127 \\
\hline Tornozelo & $24,6 \pm 3,1$ & $23,2 \pm 2,5$ & $1,4(0,7$ a 2,0$)$ & 0,001 & $24,1 \pm 2,0$ & $0,5(-0,6$ a 1,6$)$ & 0,338 \\
\hline MID & Pré & Pós & Dif(IC95\%) & & Pós & Dif(IC95\%) & \\
\hline Linha Inguinal & $68,1 \pm 7,8$ & $66,0 \pm 7,7$ & $2,1(1,3$ a 2,9$)$ & $<0,001$ & $64,8 \pm 9,9$ & $3,3(1,1$ а 1,5$)$ & 0,008 \\
\hline $7 \mathrm{~cm}$ & $63,6 \pm 8,4$ & $62,1 \pm 8,2$ & $1,4(0,5$ a 2,3$)$ & 0,005 & $60,2 \pm 9,3$ & $3,5(0,2$ a 6,7$)$ & 0,039 \\
\hline $14 \mathrm{~cm}$ & $58,6 \pm 8,0$ & $57,0 \pm 8,0$ & $1,7(1,0$ a 2,3$)$ & $<0,001$ & $54,0 \pm 8,6$ & $4,6(0,9$ а 8,3$)$ & 0,02 \\
\hline $21 \mathrm{~cm}$ & $53,6 \pm 9,0$ & $50,9 \pm 7,6$ & $2,7(0,5$ a 4,8$)$ & 0,02 & $47,8 \pm 7,6$ & $5,8(2,4$ a 9,1$)$ & 0,04 \\
\hline Linha Poplítea & $45,1 \pm 8,1$ & $44,1 \pm 8,4$ & $1,0(0,6$ a 1,4$)$ & 0,001 & $43,7 \pm 5,8$ & $1,4(-2,8$, a 5,5$)$ & 0,479 \\
\hline $7 \mathrm{~cm}$ & $37,7 \pm 4,3$ & $36,3 \pm 4,0$ & $1,4(0,4$ a 2,4$)$ & 0,012 & $36,8 \pm 4,0$ & $0,9(-0,6$ a 2,3$)$ & 0,221 \\
\hline $14 \mathrm{~cm}$ & $37,8 \pm 4,0$ & $36,5 \pm 3,9$ & $1,3(0,8$ а 1,8$)$ & $<0,001$ & $36,7 \pm 5,5$ & $1,1(-0,3$ a 2,5$)$ & 0,117 \\
\hline $21 \mathrm{~cm}$ & $33,0 \pm 4,6$ & $31,6 \pm 4,1$ & $1,5(0,9$ a 2,0$)$ & $<0,001$ & $31,3 \pm 4,1$ & $1,8(0,2$ a 3,3$)$ & 0,029 \\
\hline $28 \mathrm{~cm}$ & $27,1 \pm 3,4$ & $26,3 \pm 3,5$ & $0,8(0,1$ а 1,6$)$ & 0,035 & $26,1 \pm 3,5$ & $1,0(-0,6$ a 2,6$)$ & 0,186 \\
\hline Tornozelo & $23,9 \pm 2,4$ & $22,8 \pm 2,3$ & $1,1(0,6$ a 1,6$)$ & $<0,001$ & $23,4 \pm 1,4$ & $0,5(-0,6$ a 1,6$)$ & 0,325 \\
\hline
\end{tabular}

Houve diferença significativa tanto no efeito agudo quanto no efeito tardio em relação à dor e a fadiga nos membros inferiores após a primeira e última sessão da drenagem linfática manual (Tabela 4). 
Tabela 4. Avaliação da dor e da fadiga dos membros inferiores, antes e após a DL, efeito agudo e efeito tardio. Vitória de Santo Antão - PE. 2018.

\begin{tabular}{|c|c|c|c|c|c|c|c|}
\hline \multirow[t]{2}{*}{ Variáveis } & \multicolumn{3}{|c|}{$\begin{array}{c}\text { Efeito agudo } \\
\text { (Primeira Sessão) }\end{array}$} & \multirow{2}{*}{$\begin{array}{c}\text { Teste-t } \\
\text { pareado } \\
\text { P valor }\end{array}$} & \multicolumn{2}{|c|}{$\begin{array}{l}\text { Efeito crônico } \\
\text { (Última Sessão) }\end{array}$} & \multirow{2}{*}{$\begin{array}{r}\begin{array}{r}\text { Teste-t } \\
\text { Pareado }\end{array} \\
\text { P valor }\end{array}$} \\
\hline & Pré & Pós & Dif(IC95\%) & & Pós & Dif(IC95\%) & \\
\hline Dor & $2,6 \pm 2,0$ & $0,8 \pm 0,9$ & $1,8(0,6$ a 3,0$)$ & 0,008 & $0,9 \pm 1,1$ & $1,7(0,4$ a 3,0$)$ & 0,014 \\
\hline Fadiga & $5,1 \pm 2,8$ & $1,3 \pm 1,3$ & $3,8(1,9$ a 5,7$)$ & 0,001 & $1,5 \pm 0,9$ & $3,6(1,8$ a 5,4$)$ & 0,001 \\
\hline
\end{tabular}

Todas as voluntárias ficam muito satisfeitas com a drenagem linfática manual e recomendam o tratamento (Tabela 5).

Tabela 5. Recomendação e satisfação. Vitória de Santo Antão - PE. 2018.

\begin{tabular}{cccccccccccccc}
\hline & V 1 & V 2 & V 3 & V 4 & V 5 & V 6 & V 7 & V 8 & V 9 & V 10 \\
\hline Satisfação & +++ & +++ & +++ & +++ & +++ & +++ & +++ & +++ & +++ & +++ \\
\hline Recomendação & + & + & + & + & + & + & + & + & + & + \\
\hline
\end{tabular}

(-) Insatisfeita

(+) Pouco Satisfeita

$(++)$ Moderadamente Satisfeita

$(+++)$ Muito Satisfeita

+ Recomenda o tratamento

- Não recomenda o tratamento

\section{Discussão}

O presente estudo aborda o efeito da drenagem linfática na diminuição e prevenção de edema nos membros inferiores em gestantes no segundo e terceiro trimestre gestacional. Foram analisadas dez gestantes: quatro gestantes finalizaram com 10 sessões, duas com 7 sessões, duas com 4 sessões, uma com 6 sessões e uma com 3 sessões. Com relação ao edema gestacional, dor e fadiga nos membros inferiores, houve redução tanto na fase aguda (primeira sessão) quanto na crônica (última sessão). Todas as gestantes ficaram muito sa tisfeitas com a drenagem linfática manual e recomendariam o tratamento.

A maioria das gestantes nesse estudo desenvolveram problemas vasculares (varizes, telangectasias e edema nos membros inferiores) associados com a gestação. Esse resultado corrobora achados de um estudo transversal com 1.835 mulheres grávidas, no qual a maioria apresentavam alterações vasculares (HALL et AL., 2016). Presume-se que a gravidez seja um fator importante que contribui para o aumento da incidência de varizes em mulheres, o que, por sua vez, pode levar à insuficiência venosa e ao edema nas membros inferiores. O sintoma mais comum de varizes e edema é a dor substancial sentida, assim como cãibras noturnas, dormência, formigamento, pernas pesadas e doloridas e possivelmente, fadigadas (SMYTH, AFLAIFEL, BAMIGBOYE, 2015).

Apenas uma paciente fazia uso de meias compressivas durante toda a pesquisa. Os tratamentos de edema gestacional nos membros inferiores tem por finalidade principalmente reduzir os sintomas ao invés de curalo. O edema gestacional por ser tratar de uma disfunção vascular resultante das alterações hormonais na gra- 
videz, fica inviável a cura totalmente, pois não existe a possibilidade de controlar os hormonios gestacionais. Uma revisão da Cochrane sobre intervenções para tratamento de varizes e edema de membros inferiores na gravidez, não encontrou evidências quanto ao uso de meias compressivas e drenagem linfática manual em uma qualidade de evidência muito baixa. Não foi encontrado nenhum estudo que utilizasse apenas a drenagem linfática manual e poucos estudosavaliaram o usodas meias compressivas (SMYTH, AFLAIFEL e BAMIGBOYE, 2015).

A quantidade de sessões da drenagem linfática manual foi variada entre as gestantes. Esse raciocínio foi baseado nas recomendações de estudos de intervenção. Atualmente preferem-se estudos do tipo pragmático, ou seja, estudos no qual a metodologia seja aplicável na vida como ela é. Esse tipo de estudo busca reprodu zir ao máximo aquelas encontradas na rotina, favorecem uma avaliação da eficácia da intervenção em seu “habitat natural”, isto é, a sua efetividade. Além disso, os procedimentos simplificados, porém frutos de elaboração complexa resultam em pouca ou nenhuma alteração das rotinas de trabalho e permitem a colaboração dos profissionais, diminuindo o custo dos estudos (COUTINHO, HUF e BLOCH, 2003).

Houve redução significativa do edema nos membros inferiores das gestantes logo após a primeira sessão (fase aguda) e esse resultado permaneceu até a última sessão (fase crônica). A drenagem linfática manual é grandemente utilizada na prática clínica no segundo e terceiro trimestre gestacional, sendo indicada por médicos obstetras e realizada por fisioterapeutas (FONSECA et al., 2009). Os efeitos fisiológicos da drenagem linfática no corpo da gestante promovem o aumento e a reabsorção de proteínas, desintoxicação dos meios intersticiais, aumento da velocidade da linfa, filtração e a reabsorção de proteínas nos capilares linfáti cos (WERNER, 2008). A técnica é eficaz porque aumenta a locomoção da linfa no interior dos gânglios linfáticos fazendo com que a linfa seja drenada mais rápida, diminuindo desta forma, o edema gestacional capi lar (LEDUC e LEDUC, 2000).

Os achados na redução do edema nesse estudo vão de acordo com outros na literatura.Um estudo de caso em duas gestantes que realizou de sete a quinze sessões de drenagem linfática manual nos membros inferiores no $3^{\circ}$ trimestre gestacional obteve redução significativa no edema realizando a perimetria dos mem bros inferiores nas duas voluntárias (SILVA e BRONGHOLI, 2012). Em outro estudo de caso com uma gestante no terceiro trimestre que realizou a drenagem linfática manual nos membros inferiores, houve também uma redução do edema gestacional após as sessões (CARDOSO, 2003).

A dor e a fadiga dos membros inferiores também diminuíram em todas as gestantes. Esses dois sintomas são considerados clínicos e importantes para a tomada de decisão de realizar uma drenagem linfática manual no período gestacional. Uma série de casos com 20 gestantes mostrou que o efeito da drenagem linfática não se restringe apenas a diminuição do edema gestacional como também se mostrou eficaz na diminuição da dor e da fadiga após a realização das sessões (WERNER, 2008).

Há na literatura uma lacuna nas principais bases de dados da saúde (Medline/Pubmed, Lilacs, Biblioteca Cochrane) sobre os efeitos da drenagem linfática no edema gestacional. As evidências disponíveis são em outras populações e ainda são insuficientes e inconcludentes quanto às repercussões fisiológicas e os efeitos clínicos da drenagem linfática manual no edema gestacional.

Porém, vale ressaltar que esse estudo contribuirá para a tomada de decisão clínica, uma vez que todas as gestantes ficaram satisfeitas com a técnica e recomendariam o tratamento. Um dos tripés da saúde baseada em evidências é a escolha e preferência do paciente, associado com a evidência científica e a experiência do profissional, o que foi demonstrado no nosso estudo.

DELGADO, A. et al. Efeitos da Drenagem Linfática Manual na Diminuição do Edema de Membros Inferiores em Gestantes.
Pleiade, 13(28): 49-59, Jan./Jun., 2019 DOI: 10.32915/pleiade.v13i28.545 


\section{Conclusão}

A técnica de drenagem linfática manual se mostrou eficiente na prevenção e diminuição do edema gestacional dos membros inferiores em fase aguda e em fase tardia. Além disso, contribuiu para diminuição da dor e da fadiga dos membros inferiores, o que levou em um aumento da satisfação com a técnica e a recomendação de todas as gestantes com o tratamento. No entanto, são necessários que realizem estudos de ensaios clínicos para confirmar os reais riscos e benefícios do uso da drenagem linfática manual no edema gestacional.

\section{REFERÊNCIAS}

BAMIGBOYE, A. A.; HOFMEYR, G. J. Interventions for leg edema and varicosities in pregnancy. What evidence?. Eur J Obstet Gynecol Reprod Biol. v. 129, n. 1, p. 3-8, 2006.

CARDOSO C. M. Drenagem linfática manual no edema nos membros inferiores de uma paciente no terceiro trimestre de gestação. 2003. 17 p. Trabalho de conclusão de curso - Universidade do Sul de Santa Catarina, Tubarão.

COUTINHO, E. S. F.; HUF, G.; BLOCH, K. V. Ensaios clínicos pragmáticos: uma opção na construção de evidências em saúde. Caderno de Saúde Pública. v. 19, n. 4, p. 1189-1193, jul./ago., 2003.

EMRICH, M. L. Drenagem Linfática Manual Em Gestantes: Uma Revisão Da Literatura. Goiânia: Biblioteca Virtual de Fisioterapia em DermatoFuncional. 2013.

ESTERSON, P. S. Measurementofankle joint swellingusing a Bigure-of-8*. JournalOrthop Sports PhysTher. v. 1, n. 1, p. 51-2, 1979.

FONSECA, F. M.; PIRES, J. L. V. R.; MAGALHÃES, G. M.; PAIVA, F. A.; SOUSA, C. T; BASTOS, V. P. Estudo comparativo entre a drenagem linfática manual e atividade física em mulheres no terceiro trimestre de gestação. Fisioterapia Ser. v. 4, n. 4, 2009.

GUIRRO, E.; GUIRRO, R. Fisioterapia em estética: fundamentos, recursos e patologias. 2. ed. São Paulo: Manole, 2003.

HALL, H.; LAUCHE, R.; ADAMS, J.; STEEL, A.; BROOM, A.; SIBBRITT, D. Healthcare utilisation of pregnant women who experience sciatica, leg cramps and/or varicose veins: A cross-sectional survey of 1835 pregnant women. WomenBirth. v. 29, n. 1, p. 35-40, 2016. http://dx.doi.org/10.1016/ j.wombi.2015.07.184.

LEDUC, A.; LEDUC, O. Drenagem linfática teoria e prática. 2. ed. São Paulo: Manole, 2000.

PIMENTA, C. A. M. Escalas de avaliação de dor. In: TEIXEIRA, M.D. (ed.) Dor conceitos gerais. São Paulo: Lima. 1994, p. 46-56.

SANTOS, F. P. Análise comparativa da drenagem linfática manual e inelastoterapia aplicadas no edema gestacional em membros inferiores. 2009.

SILVA, M.D.; BRONGHOLI, K. Drenagem linfática corporal no edema gestacional. Ver Iterbio. v. 1, n. 2, 2012.

SILVA, A. C.; BARRETO, E. A. S.; FERDINANDO, J. S.; BARBOSA, M. S. T. Os benefícios da drenagem linfática em gestantes. Revista Eletrônica Belezain. 2015. 
SMYTH, RMD, AFLAIFEL, N, BAMIGBOYE, A. A. Interventions for varicose veins and leg oedema in pregnancy. Cochrane Database of Systematic Reviews. v. 10, n. CD001066. 2015. DOI: 10.1002/14651858.CD001066.pub3. 2015.

SOUZA, E. L. B. L. Fisioterapia aplicada à obstetrícia e aspectos de neonatologia: uma visão multidisciplinar. 2. ed. Belo Horizonte: Health, 2000.

WERNER, A. A. et al. Drenagem Linfática. 1. ed. São Paulo: SENAC, 2008.

WOLF, E. R.; THEISS, T. Importância da realização de drenagem linfática em gestantes. Universidade do Vale do Itajaí, Balneário Camboriú. 2011. 